\title{
La publicité en FLE : un support didactique transgressif et motivant
}

Marine ABRAHAM, Université de Murcia, Espagne

\section{Introduction}

Les affiches publicitaires représentent de nos jours un outil didactique transgressif pertinent en cours de Français Langue Étrangère (FLE). Il s'agit en effet d'un support qui motive l'apprenant, de par son caractère authentique et actuel, et qui ne respecte pas nécessairement les règles imposées de manière explicite ou implicite. Or l'individu lors de la phase adolescente a tendance à vivre dans la transgression afin de progresser dans la création de son identité. De la même manière, la publicité tend à transgresser les normes, tant socioculturelles que linguistiques, afin de rester actuelle et de répondre aux attentes des consommateurs. L'individu et, dans le cadre de notre étude, l'adolescent se sent par conséquent identifié et motivé par les affiches publicitaires, révélatrices des évolutions de nos sociétés occidentales, qui s'avèrent également être une source inépuisable de possibilités didactiques en accord avec le Cadre européen commun de référence (CECR).

\section{Des adolescents anticonformistes soumis à des normes}

\subsection{Vers de nouvelles normes}

L'adolescence, période de la vie entre l'enfance et l'âge adulte, représente une phase particulière du développement humain ponctuée de changements physiques et psychiques. Les transformations, tant somatiques que psychosociales lors de cette phase transitoire ont de fortes conséquences quant à la formation de la personnalité de l'individu. Le stade adolescent, correspondant au cinquième stade du développement humain selon Erik Erikson (98), se réfère à la période durant laquelle deux types d'identité se construisent : l'identité personnelle et l'identité sociale, cette dernière faisant référence à l'intégration du jeune dans un ou plusieurs groupes sociaux. En effet, l'adolescent cherche à créer sa propre identité avant de la soumettre aux autres individus qui font partie de son nouvel environnement. Il tente ainsi de s'intégrer à un groupe social choisi en respectant et assimilant les identités d'autrui. Par la suite, il mettra en avant la sienne, afin de se démarquer, ne serait-ce que légèrement, du reste des membres du groupe auquel il appartient désormais.

Pour ce faire, l'adolescent doit se heurter à des inadaptations passagères indispensables qui altéreront considérablement les relations qu'il entretient avec sa famille et plus amplement, les adultes. Il désire en effet se distinguer de ses parents et, ainsi, prendre ses 
distances avec les valeurs acquises durant son enfance. Le jeune remet donc en cause ces dernières qui lui ont été imposées par la société, refusant d'être normé pour devenir à son tour normatif et, ainsi, élaborer ses propres règles. Il gagne ainsi en autonomie, indispensable à la maturation psychologique nécessaire pour l'entrée dans l'âge adulte, crée de nouveaux repères qui lui sont propres et développe ainsi que diffuse sa nouvelle identité au sein de son environnement (Coslin, Psychologie, 23-27, 85).

Il ne faut cependant pas omettre le fait que la famille, certes moins influente que durant l'enfance dans le développement moral de l'individu, reste, lors de la phase adolescente, un « agent socialisateur privilégié » (Coslin, Socialisation, 19). Les conflits entre parents et adolescents sont fréquents et primordiaux, tout comme l'est la transgression de règles chez le jeune, indispensable à sa progression en tant qu'individu (28). Michel Claes fait remarquer que «durant l'enfance et l'adolescence, la vie sociale et émotionnelle se déroule surtout à l'intérieur de deux mondes : celui des relations avec les parents et celui des relations avec les pairs » (7-8). Il s'agit de ce fait de deux univers disposant de règles particulières et qui assurent des fonctions fondamentalement opposées mais complémentaires, qui permettent les développements psychologique, moral et social de l'adolescent. Les relations avec les parents se révèlent être régies par des règles d'attachement, d'autorité et d'obligations. Afin de construire sa propre identité et de se développer socialement parlant, le jeune aura tendance à se détacher émotionnellement et physiquement de l'emprise de ses parents, dans le but de se rapprocher de ses pairs avec lesquels apparaissent des règles de réciprocité, de partage d'intérêts et de valeurs ainsi que des règles de pouvoir. Ceci pourrait paraître contradictoire par rapport à ce que nous énoncions précédemment et nous amène à nous interroger sur la problématique suivante : dans quelle mesure l'adolescent serait-il à la fois normatif et normé ?

Le modèle de développement moral par stades de Lawrence Kohlberg, fortement influencé par le modèle du développement cognitif par paliers d'acquisition de J. Piaget, met en relief un changement important chez le jeune lors de sa période pubère (Cloutier et Drapeau 78). En effet les stades 3 et 4, appelés conventionnels, révèlent un besoin de conformité chez le jeune, ainsi que la nécessité de relations interpersonnelles avec des individus ayant un âge similaire au sien et partageant le plus souvent son environnement. Il intègre ainsi des règles du groupe restreint auquel il appartient et s'inquiète de ce qu'autrui peut penser de lui. Nous pourrions par conséquent décrire le jeune comme un être conformiste, adoptant une attitude passive puisqu'il ajuste ses idées et son comportement sur ceux des personnes de son milieu afin d'être approuvé par ceux-ci. 
Nous constatons que l'adolescent passe du respect de règles inculquées par sa famille au rejet de celles-ci pour finalement assimiler de nouvelles normes dictées par son groupe de pairs. Un certain paradoxe adolescent entre le conformisme et l'anticonformisme subsiste donc, tous deux nécessaires à l'affirmation de la personnalité du jeune qui transgresse des règles de son passé pour mieux accepter les nouvelles normes qui se présentent à lui.

Il s'avère que se conformer aux lois dictées par le groupe semble être indispensable afin de recevoir l'approbation sociale de ses membres. En effet, si le sujet décide d'aller à l'encontre de ces normes, il risque de susciter du mépris et pourrait se sentir rejeté. Une fois intégré, il est certain qu'il aura tendance à affirmer certains traits de caractère qui lui sont propres et ainsi prouver qu'il n'est pas une réplique et qu'il a sa propre personnalité. Néanmoins, le regard des autres, à tout âge, mais par-dessus tout pendant la phase pubère, joue un rôle primordial dans le développement social de l'individu.

\subsection{Systèmes et réseaux sociaux de l'adolescent}

Les différents systèmes sociaux

Microsystème

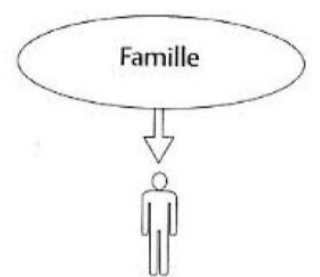

Mésosystème
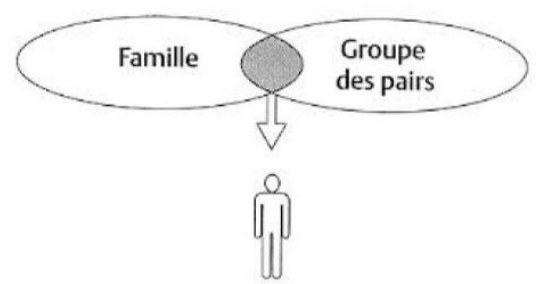

Exosystème

Famille

Groupe des pairs

Médias
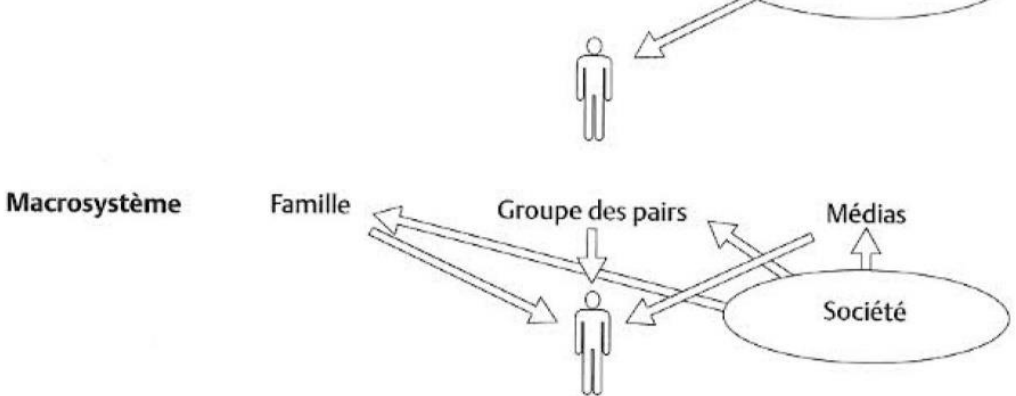

Source : Bronfenbrenner, 1979.

(Claes 36)

La famille et les pairs demeurent la base de la construction identitaire de l'adolescent. Ils sont en effet considérés comme les deux principaux systèmes sociaux apporteurs de normes, quelle que soit l'étendue du réseau social du jeune. Nous pouvons par conséquent 
nous interroger sur les lieux de rencontres de l'adolescent avec ses semblables : dans son quartier, lors d'activités sportives, etc., mais par-dessus tout, à l'école et plus tard au collège et au lycée, étant donné que son lieu de scolarité représente son deuxième agent de socialisation, après la famille. C'est donc l'endroit où il se familiarise généralement avec ses semblables. Il s'agit ici d'une autre grande contradiction : le jeune a tendance à s'éclipser du cercle familial afin de se rapprocher de ses pairs, mais il le fait au sein d'un établissement soumis à des règles strictes et où règnent les adultes. En effet, le système éducatif est soumis à une série de règles que ce soit au niveau européen avec le CECR, national avec les lois dictées par le Ministère de l'Éducation ou local avec les décrets. Au sein même de l'établissement scolaire, le règlement intérieur convient du comportement à suivre et, en ce qui concerne les cours de FLE, le département de français se réunit régulièrement pour prendre des décisions concernant le processus d'enseignement et apprentissage ainsi que les manuels à utiliser en cours de FLE.

Outre la famille et le groupe de pairs, la société est réglée par des normes implicites influencées par des principes éthiques prônant le bien commun et d'autres explicites (lois, décrets, etc.). Les moyens de communication, au service de la société de consommation, se révèlent être, pour leur part, de remarquables transmetteurs de phénomènes de mode et de normes, le plus souvent subjectives et éphémères. Dans le cas de notre étude, nous nous centrerons sur l'influence de la publicité chez le jeune. Comme souligné précédemment, le regard des autres joue un rôle prépondérant chez l'adolescent. Il est nécessairement lié au culte de l'image promu par les moyens de communication tels que la publicité, omniprésente et à laquelle le jeune est familiarisé depuis sa plus tendre enfance. Son ubiquité est telle qu'elle est devenue une préoccupation grandissante de nombreux gouvernements et organisations dans le monde entier. Plus de soixante pays en 2004 avaient déjà mis en place des dispositifs afin de réguler les annonces publicitaires destinées aux plus jeunes. L'Organisation Mondiale de la Santé ainsi que les Nations Unies ont également lancé des campagnes de prévention dans les pays occidentaux à ce sujet (Hawkes). Malgré la présence de ces mécanismes, la publicité fait véritablement partie de l'univers adolescent, de manière explicite ou implicite (Bernheim). De plus, outre sa fonction première qui est celle de promouvoir un produit afin d'en augmenter ses ventes, elle engendre des valeurs sociales et des repères devenus indispensables aux adolescents qui se retrouvent, par conséquent, sous influence constante dans leur processus de socialisation (Sapena). Nous nous rendons compte que, non seulement la publicité a une influence sur les adolescents, mais que ces derniers sont également une source d'inspiration nécessaire aux publicistes, avides de répondre aux besoins 
de reconnaissance des jeunes et de leur créer de nouvelles nécessités. Les annonceurs s'inspirent ainsi de la réalité sociale des jeunes consommateurs pour créer des affiches ou spots publicitaires et, de cette manière, leur permettre de se sentir partie intégrante de la société de consommation, en se reconnaissant dans les publicités qu'ils côtoient tous les jours (Eguizábal). La passion des jeunes pour les marques commerciales, omniprésentes dans nos médias, n'est par exemple pas anodine puisque celles-ci sont le plus souvent révélatrices de valeurs et de styles de vie adulés par les adolescents.

Pour conclure, il est important de retenir que, d'une part, l'adolescent tend à transgresser les règles qui lui sont imposées depuis l'enfance afin de se créer sa propre identité, tout en se conformant à de nouvelles normes provenant de son groupe de pairs et, respectant la plupart du temps les lois qui lui sont dictées par la société. D'autre part, nous faisons face à une standardisation du processus d'enseignement et d'apprentissage du FLE : nous remarquons en effet un «mouvement de certification et de standardisation grâce au CECR » notamment en ce qui concerne l'évaluation des acquis avec la création d'une échelle de niveau universel (Springer : 1) ainsi que l'uniformisation des compétences (Defays) qui ne laissent finalement que peu de place à la diversification pédagogique. L'acquisition de la langue française a donc tendance à être systématisée : un concept généralement assumé par le jeune. En effet, le besoin de repères, d'uniformisation et de conformité, malgré un comportement parfois désobéissant, a pour effet un entendement presque global du jeune envers les règles qui existent dans leur environnement scolaire et, par conséquent, une acceptation du système éducatif réglé. Finalement, nous remarquons que l'adolescent se montre réceptif à la publicité en général. Par conséquent, nous pouvons en déduire que si les professionnels de la publicité réussissent à séduire le jeune et à attirer son attention, pourquoi ne pas utiliser dans nos cours de FLE le résultat de leur travail ?

\section{La publicité : un outil didactique transgressif en accord avec des adolescents exigeants}

\subsection{Autonomie, authenticité et affectivité}

En dépit d'un système éducatif normé, le CECR émis par le Conseil de l'Europe en 2001 se révèle être en quelque sorte flexible et tente de répondre aux attentes adolescentes. Comme énoncé précédemment, l'individu, durant la phase pubère, devient peu à peu autonome ou du moins il tend à s'émanciper en créant une certaine distance avec ses parents, rejetant les valeurs de son passé et transgressant les règles qui lui ont été dictées. L'indépendance recherchée par le jeune est, en effet, indispensable au bon déroulement de sa 
vie socio-affective et essentielle à la création de son identité personnelle et sociale qui lui permettra son entrée dans l'âge adulte. Nous nous rendons compte que le CECR, en accord avec les besoins adolescents, préconise l'autonomie dans l'apprentissage :

Il faudrait également amener les apprenants à réfléchir sur leurs besoins en termes de communication, les entraînant ainsi à une prise de conscience de leur apprentissage et à l'autonomie. (46)

L'apprentissage autonome peut être encouragé si l'on considère «qu'apprendre à apprendre » fait partie intégrante de l'apprentissage langagier, de telle sorte que les apprenants deviennent de plus en plus conscients de leur manière d'apprendre, des choix qui leur sont offerts et de ceux qui leur conviennent le mieux. (109)

Cette dernière citation met en relief un parallélisme évident entre un processus autonome d'apprentissage et des mécanismes implicites amenant à l'émancipation socioaffective du jeune. Nous réalisons que l'évolution du jeune, tant au niveau académique que personnel, se fait en trois temps : premièrement il prend conscience de ses acquis et des valeurs qui lui ont été inculquées, pour ensuite mieux comprendre les alternatives qui se présentent à lui et, enfin, faire son choix en fonction de ce qui répond le mieux à ses intérêts, quitte à outrepasser les normes de son passé.

D'autre part, le CECR prône l'emploi de documents authentiques en cours de FLE :

En règle générale on attend des apprenants qu'ils acquièrent/apprennent une L2 selon l'une des modalités suivantes :

a. par l'exposition directe à l'utilisation authentique de la langue en L2

- en face à face avec des locuteurs natifs

- en écoutant des conversations auxquelles ils ne participent pas

- en écoutant la radio, des enregistrements, etc.

- en écoutant et regardant la télévision, des vidéos, etc.

- en lisant des textes écrits non manipulés et non progressifs (journaux, magazines, récits, romans, affiches et panneaux publics, etc.) (110-111).

Nous remarquons qu'ici aucune transformation du support didactique n'est recommandée, ce qui signifie que ce dernier se retrouve ancré dans le réel de l'apprenant. Il ne s'agit pas seulement d'un mécanisme employé en didactique du FLE puisque nous pouvons constater que les annonceurs suivent la même logique afin de capter l'attention des futurs consommateurs. Nous expliquions précédemment que les publicistes analysaient nos comportements, modes de vie, situations familiale, sociale et économique pour créer leurs affiches publicitaires. Raúl Eguizábal affirmait en effet dans son livre Industrías de la consciencia que les annonceurs s'inspiraient de notre réalité sociale et de notre quotidien pour que nous puissions nous reconnaitre dans les publicités et ainsi nous sentir identifiés. Suite à des fuites retentissantes au Baccalauréat spécialité scientifique en 2011, la célèbre 
marque de stylo Bic décida de tirer profit de ce malencontreux événement pour promouvoir son stylo phare. Situés au centre de l'image, le crayon ainsi que le slogan publicitaire «Contre les fuites au Bac : le stylo qui ne fuit pas » font clairement référence au fait divers français de l'époque, fait qui marquera les esprits adolescents durant de longs mois. Outre le caractère actuel du slogan, l'humour est également fortement présent, tournant la fâcheuse situation en dérision. De la même manière, Bic faisait la publicité de son stylo quatre couleurs pour les examens du Baccalauréat de 2011 en le mettant en scène dans quatre situations ${ }^{1}$. Quatre affiches publicitaires ont en effet été créées, chacune d'elles retransmettant une épreuve du Bac. De nouveau, l'actualité et la dérision étaient au cœur de ces campagnes publicitaires. Pour un étudiant en FLE, ces publicités lui permettent non seulement d'étudier la linguistique du slogan dans un contexte actuel mais aussi la culture française, ici son système éducatif si nous ouvrons un débat sur ce que représente le Bac pour les étudiants français par exemple. Il est par conséquent primordial de prendre en compte les goûts et besoins actuels des adolescents, que ce soit au niveau commercial, dans le cas des annonceurs, ou au niveau académique afin de motiver le jeune dans son apprentissage.

Enfin, en plus des qualités citées précédemment, les affiches publicitaires engendrent des émotions variées, souvent positives chez le jeune. D'une part, nous faisons face à un nouveau type de publicité nommé la pub choc. Étant donné l'ampleur de la violence dans nos médias ces dernières années, le monde de la publicité a vu naître ce modèle de campagnes publicitaires qui consiste à provoquer le spectateur, afin qu'il se sente touché par le message transmis. Les célèbres campagnes de prévention routière, menées depuis quelques années en France et sujettes à d'importantes controverses, illustrent ce phénomène au moyen de slogans tapageurs tels que «Vous rouliez juste un peu vite, vous l'avez juste un peu tué $»^{2}$. Des images qualifiées de choquantes voire insoutenables accompagnent ces slogans, des images qui dérogent aux règles éthiques et implicites de nos sociétés contemporaines mais qui permettent de capter instantanément l'attention de l'individu tant elles paraissent réalistes. D'autre part, l'humour demeure une stratégie intéressante qui fonctionne surtout avec les jeunes. Comme le signalent Claude Chabrol et Pierre Vrignaud, «l'humour joue clairement sur l'attention, et donc favorise une mémorisation d'éléments divers » (136), un élément notable dans les slogans publicitaires tant au niveau commercial que pour leur étude au sein d'une classe de FLE. De plus, nombreux sont ceux tels que Céline Albarracin mettant en évidence l'attrait des jeunes pour les publicités à teneur humoristique : «Lorsqu'ils se promènent sur internet, les 15-25 ans aiment s'amuser. Pour que les marques les attirent, elles doivent miser sur une relation de connivence et manier l'humour » (3). Son utilisation ne peut 
se développer qu'à partir de références socioculturelles solides. Nous revenons ici à la réalité et au quotidien de la cible visée par la publicité : si elle méconnaît le sujet à humeur positive, elle ne pourra bien évidemment pas en rire. Nous pouvons citer comme exemple d'affiche publicitaire révélatrice de ce phénomène une des publicités de la compagnie téléphonique Virgin, où le personnage principal de l'affiche représente M. Jackson, chanteur culte de la fin du 20 ème siècle, décédé en 2009 , ayant pour slogan « À ce prix-là, I'm back »³. Il s'agit ici d'une publicité reposant sur l'humour noir puisque les publicistes utilisent une personne célèbre n'étant plus de ce monde afin de vanter les mérites de leurs produits. Nous constatons que, tout comme dans les pubs chocs énoncées précédemment, la sémiotique de l'affiche s'avère être primordiale à la compréhension et au slogan, puisque sans cet apport, l'effet humoristique recherché ne pourrait exister. De plus, au niveau académique, nous nous rendons compte que les éléments sémiotiques d'une affiche publicitaire aident l'apprenant à mieux comprendre le slogan, ils lui permettront même de découvrir de nouveaux termes ou de nouvelles expressions de la langue française, tout en développant des compétences socioculturelles de manière accrue. Il est par ailleurs bon de signaler qu'en temps de crise, la connotation humoristique d'une campagne publicitaire est considérable. De plus, afin de traverser cette période difficile que représente l'adolescence, le jeune doit se sentir apaisé devant une publicité, ce doit être pour lui un divertissement. Il est démontré que l'utilisation de support didactique divertissant ou choquant permet la motivation de l'étudiant lors de son apprentissage. D'une part, l'aspect ludique d'une activité pédagogique, le plus souvent associé à la notion de jeu (Silva), concède à l'étudiant un moyen efficace de palier sa timidité et lui permet de participer de manière plus spontanée en cours de FLE. Le CECR fait d'ailleurs allusion aux activités ludiques de la langue au moyen de publicités et autres, représentatives des tâches communicatives recommandées dans les différents systèmes éducatifs européens. D'autre part, dans un monde où la violence de tout type est désormais banalisée, il s'avère généralement laborieux d'attirer l'attention du jeune. Les pubs chocs susciteront en lui un intérêt qui, la plupart du temps, engendrera un désir d'approfondir ses recherches et par la même occasion son apprentissage. C'est pour cette raison que les affiches publicitaires doivent être considérées comme un outil didactique efficace en cours de FLE.

\subsection{Une transgression des règles linguistiques françaises motivante}

Comme le souligne Antonio Ferraz Martinez dans son ouvrage El lenguaje de la publicidad, «la publicité absorbe et fait siens les langages et les moyens expressifs les plus hétérogènes dans le but de séduire les récepteurs au moyen de tout type d'armes $»^{4}(31)$. Les annonceurs ont recours à différents signes tels que l'image, le mot, la phrase, les chiffres, les 
couleurs, etc. et les remanient autant que souhaité afin d'arriver à leurs fins. Ils innovent en mélangeant par exemple divers registres (langage parlé, spécialisé, etc.), ou bien en introduisant des termes étrangers, qu'il s'agisse d'emprunts à d'autres langues ou de calques, le but étant de choquer le récepteur du message publicitaire. Ils créent finalement des néologismes ainsi que des technicismes, et transgressent de cette manière les règles de la linguistique française.

Nous tenterons de déterminer ici les raisons pour lesquelles les transgressions linguistiques auxquelles sont soumis les slogans de nos jours s'avèrent être motivantes pour les jeunes de nos sociétés contemporaines. Le concept de motivation au niveau didactique est à l'origine de nombreux ouvrages divulguant plusieurs tendances à la fois hétérogènes et complémentaires. Nous nous intéresserons ici à la théorie de la motivation intrinsèque que défendent de nombreux auteurs. Des psychologues tels que Carl Rogers soutiennent ainsi que le jeune ne doit pas nécessairement attendre de gratifications extérieures lors de la réalisation d'un travail en cours mais doit plutôt se sentir motivé par l'activité en elle-même. L'adolescent développe en effet une certaine satisfaction simplement du fait des impressions positives qu'il ressentira en prenant part à une tâche (Deci et Ryan). Denise Barbeau précise que «dans l'approche socio-cognitive, la motivation scolaire se définit comme un état qui prend son origine dans les perceptions et les conceptions qu'un élève a de lui-même et de son environnement et qui l'incite à s'engager, à participer et à persister dans une tâche scolaire » (20). En d'autres termes, de par son entourage et le climat dans lequel il développe sa personnalité, l'individu sera à même de trouver ses propres motivations afin de persévérer dans son apprentissage. Selon le Centre national de ressources textuelles et lexicales informatisé (CNTRL), l'action de motiver est « en psychopédagogie, l'ensemble des facteurs dynamiques qui suscitent chez un élève ou un groupe d'élèves le désir d'apprendre » $(<$ http://www.cnrtl.fr/definition/motivation $>$ ). Enfin, le CECR mentionne à plusieurs reprises la nécessité pour les enseignants de proposer des tâches motivantes et ancrées dans le réel des apprenants afin de susciter un intérêt chez eux et de les inciter à s'impliquer dans leur processus d'apprentissage de manière efficace.

La publicité, partie intégrante du quotidien des apprenants et reflet du monde dans lequel nous progressons, se révèle donc être un support didactique pertinent et motivant pour les jeunes de nos sociétés occidentales. Prenons comme modèle de transgressions un produit publicitaire datant de 2010 faisant la réclame d'un nouveau forfait d'une entreprise de télécommunication française au moyen d'un slogan plurilingue : «nouveau forfait mobile Origami style : pokez, taggez, likez $»^{5}$ : 
- «mobile» est le premier terme anglais recensé ci-contre. Il est pourtant question d'une expression française mais le sens qui lui est donné sur l'affiche est l'anglais ${ }^{6}$. De ce fait, le terme anglais signifie téléphone portable alors que le français a une toute autre signification : plus souvent utilisé en qualité d'adjectif que de substantif, «mobile » reflète une chose ou une personne en mouvement. Néanmoins il s'agit d'un terme compris de tous dans ce slogan et non pas en raison du contexte de son emploi mais parce qu'il est devenu, ces dernière années, un terme générique adopté par d'autres entreprises de télécommunication. Il est important de signaler sa connotation positive : « mobile » transmet en effet une impression de mouvement, le produit apparaît donc être un forfait qui pourrait s'adapter à n'importe quel consommateur.

- Avec l'expression « Origami style », on se rend compte que «style » est un mot français employé dans un système syntaxique anglais : «Origami» serait l'adjectif et « style » le substantif. Ce dernier est placé en seconde position alors que les règles du français préconisent l'inverse. «Origami » n'est d'ailleurs pas un adjectif puisqu'il s'agit d'un nom japonais faisant référence à l'art du pliage de papier, celui-ci représenté sur l'image, illustrée de personnages en papier de motifs variés. Les consommateurs de ce forfait pourront aussi voir dans l'expression « Origami style » un marqueur culturel notable quelques années plus tard : elle s'avérera être le titre d'une célèbre chanson de l'artiste Psy lancée en 2012 qui fut un succès mondial, donnant lieu à de nombreuses parodies ainsi qu'à l'apparition des flash mobs $^{7}$ organisés dans le monde entier. Cette chorégraphie insolite est de ce fait représentée sur l'image où les personnages en papier donnent l'impression d'être en train de danser. Ces diverses références culturelles servent à donner un sentiment de mouvement, d'agitation, d'effervescence au jeune. Elles transmettent l'idée que ce qui est publicité peut s'adapter aux besoins de chacun, qu'il ne s'agit pas d'un forfait fixe ou immobile, mais qu'il s'avère plutôt être souple et modifiable.

- « pokez, taggez, likez » est issu du langage employé sur le réseau social Facebook. Il est question de termes francisés étant donné la transformation des verbes anglais en verbes français du premier groupe à la deuxième personne du pluriel de l'impératif. Nous distinguons ici un clair exemple de personnification du slogan pour que le futur consommateur se sente impliqué dans la publicité, qu'il se reconnaisse. Ce slogan plurilingue est bien évidemment traduit en bas de l'affiche par «pokez, marquez, appréciez », comme requis par la loi de $1994^{8}$, mais nous nous rendrons compte que le jeune comprendra bien plus le slogan originel que sa traduction.

Les effets du plurilinguisme chez le jeune individu sont variés. Ce type de slogan, de 
plus en plus courant dans notre environnement, réunit la plupart des intérêts adolescents et, par la même occasion, réussit à le motiver en cours de FLE. Premièrement, il réussit à choquer le jeune; ce dernier se sent interpellé par cet ensemble de termes aux sonorités étrangères mais peu lointaines. Il représente également un pays producteur et est régulièrement synonyme d'excellence pour l'adolescent. Comme nous le signale Ferraz Martinez, «Avec les références faites aux pays et régions, les produits peuvent avoir une connotation positive. Nous pouvons donc profiter ou bien du prestige de ce qui est étranger, ou des arguments nationalistes, ou des références à des milieux supranationaux ${ }^{9}$ (47). Ensuite, le slogan plurilingue est un indicateur d'internationalisme : il procure une sensation d'ouverture sur le monde que le jeune recherche ces dernières années (Lemaire). Nous pourrions même aller plus loin en affirmant que l'emploi de langues étrangères dans le slogan peut aussi amener à une impression de prestige social ou intellectuel puisque le jeune, en mesure de comprendre ce slogan si particulier, se rend compte de capacités siennes encore méconnues (Lemaire). Pour toutes ces raisons, les affiches publicitaires représentent un support didactique adapté aux étudiants de FLE, grâce à leurs slogans répondant le plus souvent aux attentes du CECR. L'approche plurilingue s'inscrit, en effet, dans les compétences émises par le CECR, suite à l'évolution de l'Europe vers une société multilingue et multiculturelle :

Il faut resituer le plurilinguisme dans le contexte du pluriculturalisme. La langue n'est pas seulement une donnée essentielle de la culture, c'est aussi un moyen d'accès aux manifestations de la culture. L'essentiel de ce qui est énoncé ci-dessus s'applique également au domaine le plus général. Les différentes cultures (nationale, régionale, sociale) auxquelles quelqu'un a accédé ne coexistent pas simplement côte à côte dans sa compétence culturelle. Elles se comparent, s'opposent et interagissent activement pour produire une compétence pluriculturelle enrichie et intégrée dont la compétence plurilingue est l'une des composantes, elle-même interagissant avec d'autres composantes. (12)

D'autre part, nous remarquons que le slogan, défiant les règles de la langue française que ce soit au niveau syntaxique, lexical, ou grammatical, afin de motiver l'adolescent, fait l'usage d'une langue de registre plus familier que formel et, le plus souvent, use de la langue anglaise sous toutes ses formes. L'anglais comme langue dominante se révèle être une manifestation sociolinguistique d'ordre global étant donné qu'il se diffuse par lui-même, à travers les médias et, de manière générale, au sein de notre environnement social, culturel et économique. Selon de nombreux linguistes tels que Gilles Fauconnier, la connaissance de la langue provient de l'usage de celle-ci. Or le franglais est considéré comme la langue des 
adolescents et ce depuis la deuxième moitié du vingtième siècle (Étiemble), de par son caractère insolite, expressif, évolutif, créatif et par-dessus tout transgressif. Le jeune Français se délecte donc de l'usage du franglais. De plus, notre quotidien se trouve dès lors régi par les nouvelles technologies, dévotes de la langue anglaise (Maurais) : l'OCDE est d'ailleurs à l'origine d'un rapport à ce sujet et conclut que « l'anglais est de loin la langue du commerce électronique » (228). La publicité, consciente de ces transformations sociolinguistiques utilise donc ce «vocabulaire et les valeurs émergeant chez les ados» (Dagnaud 42). Le slogan plurilingue répond donc aux attentes du jeune adolescent et permet, par conséquent que les affiches publicitaires représentent un support didactique notable en cours de FLE.

Cependant, nous pourrions nous interroger sur les bienfaits de ces transformations linguistiques transgressives dans l'apprentissage d'un étudiant en FLE. En effet, ces slogans vont bien souvent à l'encontre des règles de la langue française que le jeune est censé étudier en cours de langue. Néanmoins, de plus en plus, les enseignants choisissent ce type de support de par les possibilités pédagogiques que celui-ci engendre. L'étude d'affiches publicitaires amène effectivement l'élève à développer de nombreuses compétences didactiques telles que la compétence communicative (description à l'oral de l'image ou émission d'hypothèses sur le contenu de l'affiche) ou la compétence linguistique, bien que cette dernière puisse paraitre complexe à développer. Prenons le cas du slogan plurilingue suivant : «Tchatez avec vos amis où que vous soyez $»^{10}$, où le premier terme se révèle être un mot anglais francisé. Il est certain que le verbe «tchater », n'étant pas à proprement parler issu du français (construit à partir du verbe « to chat » en anglais, ayant le sens de bavarder), ce qui nous intéressera seront les autres éléments linguistiques du slogan qui nous permettent, par exemple, de montrer au jeune un aperçu de l'utilisation du subjonctif de manière implicite et ludique. Enfin, il est important de préciser que de nombreuses affiches publicitaires actuelles usent de moyens linguistiques autres que plurilingues qui permettent un enseignement distinct, tel le slogan suivant: «N'en perds pas une miette, finis ton assiette. ${ }^{11}{ }^{1}$, grâce auquel il est possible de faire l'étude de la formation de l'impératif, tant à l'affirmatif qu'à la forme négative, ou dans celui-ci «Qui jette un œuf, jette un bœuf $»^{12}$, un détournement de proverbe qui amène l'étudiant à s'interroger sur les figures de style françaises.

\section{Conclusion}

Pour conclure, nous retiendrons de cette étude l'aspect motivant du support didactique que représentent les affiches publicitaires et les enseignements que celles-ci profèrent aux 
étudiants de FLE, malgré et, par-dessus tout, grâce à leur caractère transgressif. Ainsi, au niveau académique, les campagnes publicitaires permettent une étude ludique de la langue française, suivant les évolutions linguistiques et culturelles de nos sociétés occidentales. Les élèves de FLE sont en effet à même de découvrir la culture française et ses valeurs socioculturelles actuelles à partir des thèmes transversaux traités dans les affiches publicitaires, telles que les publicités sociétales citées antérieurement contre le gaspillage alimentaire. Ce document authentique engendre également de nombreux apprentissages personnels, puisqu'il permet à l'adolescent de prendre conscience des répercussions de la publicité ou, du moins, de se familiariser avec les techniques commerciales utilisées par les annonceurs afin de vendre leurs produits et de fidéliser leur clientèle. En effet, les débats que peuvent engendrer en cours de FLE l'analyse de ce type de document amènent le jeune à prendre connaissance des outils existant pour analyser les effets des campagnes publicitaires. Il s'agit, en quelque sorte, d'un moyen de prévention face aux travers de nos sociétés de consommation.

\section{Bibliographie}

Albarracin, Céline. « Séduire et fidéliser la génération Y, un enjeu majeur pour les marques ». 2014. http://blog.alerti.com/fr/2014/04/seduire-fideliser-generation-y/. 05/01/2015. Barbeau, Denise. « La motivation scolaire ». Pédagogie collégiale 7 : 1 (1993) : 20-27. Bernheim, François. Guide de la publicité et de la communication. Paris : Larousse, 2004.

Chabrol, Claude et Pierre Vrignaud. «Enquête psycho-langagière sur les effets de 1'humour en publicité ». Questions de communication $10 \quad$ (2006). [http://questionsdecommunication.revues.org/7695]. 03/09/2013.

Claes, Michel. L'expérience adolescente. Liège : Mardaga, 1991.

Cloutier, Richard et Sylvie Drapeau. Psychologie de l'adolescence. 3e éd. Montréal : Gaëtan Morin, 2008.

Conseil de l'Europe. Cadre européen commun de référence pour les langues. 2001. [http://www.coe.int/t/dg4/linguistic/CADRE_FR.asp]. 13/04/2010.

Coslin, Pierre. La socialisation de l'adolescent. Paris : Armand Colin, 2007.

---. Psychologie de l'adolescent. 4e éd. Paris : Armand Colin, 2013.

Dagnaud, Monique. Enfants, consommation et publicité télévisée. Paris : Documentation française, 2003.

Deci, Edward et Richard Ryan. Intrinsic Motivation and Self-determination in Human 
Behaviour. New York : Plenum, 1985.

Defays, Jean-Marc. « Enseignant de langues : de 1'homme-orchestre au chef d'orchestre. État des lieux d'une discipline et d'une profession en questionnement ». L'enseignant au sein du dispositif d'enseignement/apprentissage de la langue étrangère. Dir. MarieThérèse Maurer-Feder. Besançon : ADCUEFE, 2013. 11-20.

Eguizábal, Raúl. Industrías de la consciencia : una historia social de la publicidad en España (1975-2009). Barcelone : Península, 2009.

Erikson, Erik. Adolescence en crise : la quête de l'identité. Paris : Flammarion, 1968.

Étiemble, René. Parlez-vous franglais ? Paris : Gallimard, 1964.

Fauconnier, Gilles. Espaces mentaux : aspects de la construction du sens dans les langues naturelles. Paris : Minuit, 1986.

Ferraz Martinez, Antonio. El lenguaje de la publicidad. Madrid : Arco Libros, 1995.

Hawkes, Corinna. Marketing Food to Children: The Global Regulatory Environment. Genève : WHO, 2004.

Kohlberg, Lawrence. The Psychology of Moral Development. San Francisco : Harper et Row, 1984.

Lemaire, Stéphanie. Le plurilinguisme dans la publicité. Göttingen : Cuvillier, 2000.

Maurais, Jacques et al. «Le français dans les nouvelles technologies de l'information ». L'avenir du français. Paris : Archives contemporaines, 2008. 49-52.

OCDE. Perspectives des technologies de l'information de l'OCDE : Les TIC et l'économie de l'information. Paris : OCDE, 2002.

Rogers, Carl. Liberté pour apprendre. Paris : Dunod, 1984.

Sapena, Nathalie. L'enfant jackpot. Paris : Flammarion, 2005.

Silva, Haydée. «La créativité associée au jeu en classe de Français Langue Étrangère ». Synergie Europe 4 (2009) : 105-117.

Springer, Claude. «Le CECR : outil de diversification ou de standardisation? ». Actes du Colloque international « Cadre Européen Commun de Référence et Enseignement $d u$ Français en Corée» de la Société Coréenne d'Enseignement de Langue et de Littérature Françaises. Séoul, 2011.

[http://springcloogle.blogspot.com.es/2011/10/cecr-standardisation-oudiversification.html]. 05/01/2015. 
NOTES

$1 \mathrm{http} / / / w w w . l e s m a r q u e s e t m o i . f r / 2011 / 04 / 23 /$ le-bic-se-met-en-4-couleurs-pour-le-bac/ [consulté le 08/08/2014]. 2 http://ecs-paris.com/blogs/digicom-2012/medias/securite-routiere-le-trash-pour-reveiller-les-consciences [consulté le 09/08/2014].

3 http://www.ozap.com/actu/pub-virgin-mobile-se-moque-de-gerard-depardieu/445664 [consulté le $06 / 05 / 2013]$.

4 Traduction personnelle de «la publicidad absorbe y hace suyos los más heterogéneos lenguajes y medios expresivos en su afán por conquistar a los receptores con todo tipo de armas ».

5 http://francebienvenue2.wordpress.com/2010/11/21/je-poke-tu-tagges-il-like/ [consulté le 26/08/2013].

6 Selon le CNTRL, un mobile est un "espace que l'on peut modifier [...] dont l'apparence donne une impression de souplesse, de mouvement, de changement » (<http://www.cnrtl.fr/definition/mobile/substantif $\rangle$ [consulté le 03/08/2013]).

7 Une flash mob est le rassemblement éclair d'un groupe de personnes dans un lieu public pour y effectuer des actions convenues d'avance, avant de se disperser rapidement parmi la foule. Elle est généralement organisée au moyen d'internet et les participants ne se connaissent pour la plupart pas.

8 Loi $N^{\circ} 94 / 665$ du 4 août 1994 relative à l'emploi de la langue française, nommée loi Toubon. http://www.legifrance.gouv.fr/affichTexte.do?cidTexte=LEGITEXT000005616341\&dateTexte=vig [consulté le 05/01/2015].

9 Traduction personnelle de «Con las referencias a países y regiones se pueden connotar positivamente los productos. Entonces se puede sacar partido bien del prestigio de lo extranjero, bien de argumentos nacionalistas, bien de referencias a ámbitos supranacionales ».

10 http://www.chunkybuzz.com/2009_11_01_archive.html [consulté le 26/08/2013].

$11 \mathrm{http}: / /$ www.al-kanz.org/2013/02/03/gaspillage-alimentaire/ [consulté le 22/04/2013].

$12 \mathrm{http}: / /$ www.al-kanz.org/2013/02/03/gaspillage-alimentaire/ [consulté le 22/04/2013]. 